\title{
A Nonlinear Time-Domain Homogenization Technique for Laminated Iron Cores in Three-Dimensional Finite Element Models
}

\author{
J. Gyselinck, R. V. Sabariego and P. Dular
}

\begin{abstract}
The authors present a novel nonlinear homogenization technique for laminated iron cores in 3D FE models of electromagnetic devices. It takes into account the eddy current effects in the stacked core without the need of modelling all laminations separately. A nonlinear constitutive magnetic law is considered. The system of nonlinear algebraic equations obtained after time discretisation is solved by means of the NewtonRaphson scheme. By way of validation the method is applied to a 3D FE model of a laminated ring core with toroidal coil.
\end{abstract}

\section{INTRODUCTION}

The magnetic cores of electromagnetic AC devices are often laminated as this is an efficient measure for reducing the eddy current losses due to the time-varying flux. As a result, the eddy current losses, and the iron losses as a whole, have a limited effect on the device characteristics; they obviously affect the overall losses and thus the efficiency of the device, but to a lesser extent e.g. the torque output. Accordingly, in 2D or 3D FE calculations, the stacked iron core is commonly assumed nonconducting and homogeneous (i.e. not laminated), and a nonlinear single-valued BH-curve is adopted. Based on the FE results, i.e. the time and space distribution of the induction in the iron core, an estimation of the iron losses may be obtained a-posteriori [1]. The FE analysis may be enhanced by considering a more involved constitutive law for the homogenized iron. A static or dynamic hysteresis model may be adopted, along with a 1D low-frequency eddy current model, in which skin and edge effects in the laminations are neglected [2].

In some applications the eddy currents in laminated iron cores may considerably alter the overall behavior of the device under study. This may be the case in power electronic applications where working frequencies and harmonic distortion are constantly increasing. Finely discretizing each separate lamination in a FE modelling is mostly out of the question. Such a brute-force approach would indeed result in huge memory requirements and calculation times. Dedicated homogenization methods are thus indispensable.

Manuscript received November 11, 2005. The research was partly carried out in the frame of the Inter-University Attraction Pole IAP P5/34 for fundamental research funded by the Belgian federal government.

The first author is with the Dept. of Electrical Engineering, Universite Libre de Bruxelles (ULB), Belgium (johan.gyselinck@ulb.ac.be). The second and third author are with the Dept. of Electrical Engineering, University of Liège, Belgium. P. Dular is a Research Associate with the Belgian National Fund for Scientific Research (F.N.R.S.).
In a frequency-domain FE analysis, considering nonsaturated iron, a complex reluctivity may be adopted in the homogenized core [3]. The frequency-dependent complex value follows from a 1D lamination model including skin effect [5]. This can be extended to the time domain by introducing skin-effect basis functions and additional degrees of freedom in the iron core [4]. Both approaches are limited to a linear magnetic material. In view of the inherent magnetic saturation of many electromagnetic apparatus, nonlinear methods are of more practical interest. In this paper the time-domain homogenization method is extended to the nonlinear case. After a brief outline of the method, it is validated by means of a 3D test case.

\section{TIME-DOMAIN HOMOGENIZATION}

\section{A. ID model of a lamination \& skin effect basis functions}

We consider a lamination of thickness $d(-d / 2 \leq z \leq d / 2)$ that carries a magnetic induction $b(z, t)$, along e.g. the $x$ axis, as shown in Fig. 1. A homogeneous isotropic material of conductivity $\sigma$ is assumed. The magnetic field $h(z, t)$, equally along the $x$-axis, is linked to the induction $b(z, t)$ by the reversible constitutive law $h=h_{f e}(b)$. The current density $j(z, t)$ is along the $y$-axis. Imposing a nonzero net flux and a zero current, the following symmetries hold: $b(z, t)=$ $b(-z, t), h(z, t)=h(-z, t)$ and $j(z, t)=-j(-z, t)$. The $1 \mathrm{D}$ eddy current problem is governed by the following partial differential equation:

$$
\frac{\partial^{2} h}{\partial z^{2}}=\sigma \frac{\partial b}{\partial t} \quad \text { with } \quad h=h_{f e}(b) .
$$

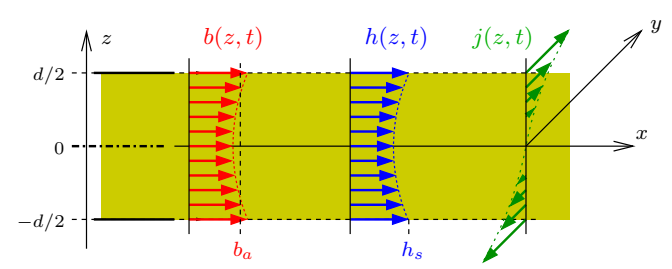

Fig. 1. Variation of $b, h$ and $j$ throughout lamination thickness

Relevant to the homogenization are the induction averaged over the thickness, $b_{a}(t)=\frac{1}{d} \int_{-d / 2}^{d / 2} b(z, t) d z$, and the surface magnetic field $h_{s}(t)=h(z= \pm d / 2, t)$.

The resolution of (1) can be developed either in the frequency domain [3], [6] or the time domain [2], [3], [6]. The frequency-domain approach is limited to linear materials, having a constant permeability $\mu_{f e}=b / h$ or constant reluctivity 
$\nu_{f e}=h / b$, whereas the time-domain approach is applicable to nonlinear materials as well.

In the frequency domain, considering a sinusoidal $h_{s}(t)$ and $b_{a}(t)$ of frequency $f$ (or pulsation $\omega=2 \pi f$ ), the equivalent complex reluctivity $\boldsymbol{\nu}_{f e}=\boldsymbol{h}_{s} / \boldsymbol{b}_{a}$, with $\boldsymbol{h}_{s}$ and $\boldsymbol{b}_{a}$ the complex representation of the $h_{s}(t)$ and $b_{a}(t)$, has the following analytical expression:

$\boldsymbol{\nu}_{f e}=\nu_{f e} \frac{d / \delta}{2}\left(\frac{\sinh d / \delta+\sin d / \delta}{\cosh d / \delta-\cos d / \delta}+\jmath \frac{\sinh d / \delta-\sin d / \delta}{\cosh d / \delta-\cos d / \delta}\right)$,

where $\jmath$ is the imaginary unit, and $\delta=\sqrt{2 / \sigma \mu_{f e} \omega}$ the penetration depth [5]. This complex reluctivity is straightforwardly adopted in a FE analysis, without any additional computational cost. Its imaginary part effects the eddy current losses.

An approximate time-domain solution of (1) can be obtained with a 1D FE model, possibly considering involved constitutive laws [6]. In order to minimize the number of spatial degrees of freedom, polynomial basis functions that are nonzero over the complete interval $-d / 2 \leq z \leq d / 2$ are introduced for interpolating $b(z, t)$ and $h(z, t)$; these are the so-called skin effect basis functions.

The starting point is the expansion of $b(z, t)$ :

$$
b(z, t)=\alpha_{0}(z) b_{a}(t)+\alpha_{2}(z) b_{2}(t)+\ldots,
$$

where the even polynomial basis functions $\alpha_{0}(z)=1, \alpha_{2}(z)=$ $-\frac{1}{2}+6(z / d)^{2}, \ldots$, are orthogonal, $\frac{1}{d} \int_{-d / 2}^{d / 2} \alpha_{i}(z) \alpha_{j}(z) d z=$ 0 if $i \neq j$, and have unit value on the lamination surface, $\alpha_{i}\left(z= \pm \frac{d}{2}\right)=1$.

The magnetic field $h(z, t)$ is subsequently expanded considering the surface magnetic field $h_{s}(t)$ and the even polynomial basis functions $\beta_{k}(z)$ of order $k \geq 2$ :

$$
h(z, t)=h_{s}(t)-\sigma d^{2} \beta_{2}(z) \frac{d b_{a}}{d t}-\sigma d^{2} \beta_{4}(z) \frac{d b_{2}}{d t}-\ldots,
$$

where the latter are zero on the lamination surface, $\beta_{i}(z=$ $\left.\pm \frac{d}{2}\right)=0$, and further determined so that $b(z, t)$ and $h(z, t)$ satisfy (1) identically, i.e. $\frac{d^{2} \beta_{i}}{d z^{2}}=-\alpha_{i-2}(z) / d^{2}$. These conditions produce then for $k$ equal to 2 and $4: \beta_{2}(z)=\frac{1}{8}-\frac{1}{2}(z / d)^{2}$ and $\beta_{4}(z)=-\frac{1}{32}+\frac{1}{4}(z / d)^{2}-\frac{1}{2}(z / d)^{4}$ respectively.

When considering a finite number of basis functions, up to order $n$ for $b(z, t)$ and order $n+2$ for $h(z, t)$, the constitutive law $h=h_{f e}(b)$, whether linear or nonlinear, cannot be satisfied identically. It can be weakly imposed as follows:

$$
\frac{1}{d} \int_{-d / 2}^{d / 2}\left(h(z, t)-h_{f e}(b(z, t)) \alpha_{k}(z) d z=0,\right.
$$

which leads to $1+n / 2$ equations $(k=0,2, \ldots, n)$ in terms of $b_{a}(t), b_{2}(t), \ldots, b_{n}(t)$, and $h_{s}(t)$. For instance, for the linear case with $n=2$, a system of two linear differential equations is obtained:

$$
\left[\begin{array}{c}
h_{s} \\
0
\end{array}\right]=\nu_{f e}\left[\begin{array}{ll}
1 & 0 \\
0 & \frac{1}{5}
\end{array}\right]\left[\begin{array}{l}
b_{a} \\
b_{2}
\end{array}\right]+\frac{\sigma d^{2}}{420}\left[\begin{array}{rr}
35 & -7 \\
-7 & 2
\end{array}\right] \frac{d}{d t}\left[\begin{array}{l}
b_{a} \\
b_{2}
\end{array}\right],
$$

with either $h_{s}(t)$ or $b_{a}(t)$ given function of time. By means of e.g. the so-called $\theta$-scheme, which amounts to the backward Euler and Crank-Nicolson scheme with $\theta$ equal to 1 and 0.5 respectively, a system of algebraic equations is obtained for each time step from $t_{i}$ to $t_{i+1}=t_{i}+\Delta t$.

For a given maximum error, the order $n$ of the spatial interpolation of $b(z, t)$ has to been increased along with the frequency $f$ (or the relative lamination thickness $d / \delta$ ). Allowing a $1 \%$ maximum error on the equivalent complex reluctivity (2), a constant interpolation $(n=0)$ is valid up to roughly $d / \delta=1$. Adding one or two interpolation functions ( $n=2$ and $n=4$ resp.) extends the validity range to $d / \delta$ equal to 4 and 8 respectively [4].

In the nonlinear case with $n=2$, the system (5) becomes

$$
\left[\begin{array}{c}
h_{s} \\
0
\end{array}\right]=\frac{\sigma d^{2}}{420}\left[\begin{array}{rr}
35-7 \\
-7 & 2
\end{array}\right] \frac{d}{d t}\left[\begin{array}{l}
b_{a} \\
b_{2}
\end{array}\right]+\frac{1}{d} \int_{-d / 2}^{d / 2} h_{f e}(b(z, t))\left[\begin{array}{l}
\alpha_{0} \\
\alpha_{2}
\end{array}\right] d z,
$$

with $b(z, t)=b_{a}(t)+\alpha_{2}(z) b_{2}(t)$. The nonlinear algebraic equations that result from the time discretization can be solved by means of the Newton-Raphson method. With given $h_{s}(t)$, the Jacobian matrix reads

$$
\frac{\sigma d^{2}}{420 \Delta t}\left[\begin{array}{cc}
35 & -7 \\
-7 & 2
\end{array}\right]+\frac{\theta}{d} \int_{-d / 2}^{d / 2} \frac{d h_{f e}}{d b}\left[\begin{array}{cc}
\alpha_{0} \alpha_{0} & \alpha_{0} \alpha_{2} \\
\alpha_{2} \alpha_{0} & \alpha_{2} \alpha_{2}
\end{array}\right] d z,
$$

where $\frac{d h_{f e}}{d b}$ is the differential reluctivity. The integration in the interval $[0, d / 2]$, exploiting the symmetry with respect to $z=0$, can be done numerically, by means of e.g. the 5-point Gauss scheme for the case $n=2$.

\section{B. FE implementation}

We consider an eddy current problem in a 3D domain $\Omega$ and its formulation in terms of the magnetic vector potential $\underline{a}$. The induction $\underline{b}=\operatorname{curl} \underline{a}$ and the electrical field $\underline{e}=-\partial_{t} \underline{a}$ thus satisfy $\operatorname{div} \underline{b}=0$ and curl $\underline{e}=-\partial_{t} \underline{b}$. The current density $\underline{j}$ is imposed in a subdomain $\Omega_{s}$ and induced in another conducting subdomain $\Omega_{l}$. The vector potential is e.g. discretised by means of edge basis function $\underline{\gamma}_{j}: \underline{a}=\sum_{j} a_{j} \underline{\gamma}_{j}$. The weak form of Ampère's law curl $\underline{h}=\underline{j}$ produces as many equations as degrees of freedom $a_{j}$ :

$$
\left(\underline{h}(\operatorname{curl} \underline{a}), \operatorname{curl} \underline{\gamma}_{i}\right)_{\Omega}+\left(\sigma \partial_{t} \underline{a}, \underline{\gamma}_{i}\right)_{\Omega_{l}}=\left(\underline{j}, \underline{\gamma}_{i}\right)_{\Omega_{s}},
$$

where $\underline{h}=\underline{h}(\underline{b})$ is the vector constitutive law in $\Omega ;(\cdot, \cdot)_{\Omega}$ denotes the volume integral in $\Omega$ of the scalar product of the two vector arguments. The uniqueness of $\underline{a}$ can be ensured by considering an edge co-tree in the nonconducting domain $\Omega \backslash \Omega_{l}$.

We consider the case where $\Omega_{l}$ consists of laminations of a stacked iron core. The insulating layers between the laminations are assumed to be negligibly thin (near $100 \%$ fill factor). We will further assume that the $\underline{h}$ and $\underline{b}$ vectors are parallel to the plane of the laminations, as is the case in the axisymmetric application example below. (See [4] for a more complete analysis, including non-negligible insulation layers and perpendicular flux.) If the lamination thickness $d$ is small compared to the overall dimensions of the core, the surface magnetic field $\underline{h}_{s}$ and the average induction $\underline{b}_{a}$ vary little from one lamination to the next. On the basis of these quantities, continuous slowly-varying fields can be defined in every point of the core volume $\Omega_{l}$; the latter will be denoted by $\underline{h}_{s}$ and 
$\underline{b}_{a}$ as well. According to the 1D lamination model presented above, $\underline{h}_{s}$ depends on $\underline{b}_{a}$ and induction components $\underline{b}_{2}, \underline{b}_{4}$, $\ldots$, the latter being equally the continuus vector extension of the components $b_{2}, b_{4}, \ldots$ introduced above.

The weak form (9) can be simplified by considering $\underline{h}_{s}$ :

$$
\left(\underline{h}(\operatorname{curl} \underline{a}), \operatorname{curl} \underline{\gamma}_{i}\right)_{\Omega \backslash \Omega_{l}}+\left(\underline{h}_{s}, \operatorname{curl} \underline{\gamma}_{i}\right)_{\Omega_{l}}=\left(\underline{j}_{i} \underline{\gamma}_{i}\right)_{\Omega_{s}},
$$

as this amounts to considering for Ampère's integral law, contours $C$ that are not linked with the induced current density in the laminations (see Fig. 3). The eddy current term produced by $\Omega_{l}$ in (9) thus vanishes.

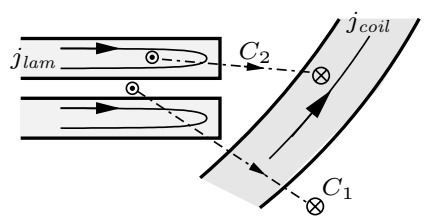

Fig. 2. Contours for Ampère's integral law: contour $C_{1}$ is linked with the current in the coil $\left(j_{\text {coil }}\right)$ but not with the eddy currents in the laminations ( $j_{\text {lam }}$, resulting in zero net current parallel to the laminations), whereas contour $C_{2}$ is linked with both

The induction $\underline{b}$ in $\Omega \backslash \Omega_{l}$ and the average induction $\underline{b}_{a}$ in $\Omega_{l}$ will be derived from the vector potential $\underline{a}$ and its edge basis function interpolation $\underline{a}=\sum_{j} a_{j} \underline{\gamma}_{j}$ defined in the whole domain $\Omega$ (with tree gauging throughout). This guarantees the continuity of the average normal induction component at the boundary of $\Omega_{l}$. The additional quantities $\underline{b}_{2}, \underline{b}_{4}, \ldots$ in $\Omega_{l}$ may be interpolated with vector basis functions that are elementwise constant and are directed in two perpendicular directions in the plane of the laminations; these basis functions are further denoted by $\underline{\zeta}_{i}$.

We consider first the linear case, with constant reluctivity $\nu$ in $\Omega \backslash \Omega_{l}$ and $\nu_{f e}$ in $\Omega_{l}$, and with $n=2$. Using the first line of (6), the equation (10) becomes

$$
\begin{gathered}
\left(\nu \operatorname{curl} \underline{a}, \operatorname{curl} \underline{\gamma}_{i}\right)_{\Omega \backslash \Omega_{l}}+\left(\nu_{f e} \operatorname{curl} \underline{a}, \operatorname{curl} \underline{\gamma}_{i}\right)_{\Omega_{l}}+ \\
\left(\frac{\sigma d^{2}}{12} \partial_{t} \operatorname{curl} \underline{a}, \operatorname{curl} \underline{\gamma}_{i}\right)_{\Omega_{l}}-\left(\frac{\sigma d^{2}}{60} \partial_{t} \underline{b}_{2}, \operatorname{curl} \underline{\gamma}_{i}\right)_{\Omega_{l}}=\left(\underline{j}, \underline{\gamma}_{i}\right)_{\Omega_{s}} .
\end{gathered}
$$

The second line of (6) is weakly imposed in $\Omega_{l}$ considering each of the basis functions $\underline{\zeta}_{i}$ :

$$
\left(\frac{\nu}{5} \underline{b}_{2}, \underline{\zeta}_{i}\right)_{\Omega_{l}}-\left(\frac{\sigma d^{2}}{60} \partial_{t} \operatorname{curl} \underline{a}, \underline{\zeta}_{i}\right)_{\Omega_{l}}+\left(\frac{\sigma d^{2}}{210} \partial_{t} \underline{b}_{2}, \underline{\zeta}_{i}\right)_{\Omega_{l}}=0 .
$$

Let us considering now a nonlinear relation $\underline{h}=\underline{h}_{f e}(\underline{b})$ between the local vectors $\underline{b}$ and $\underline{h}$ in the laminations, with $\underline{b}=\alpha_{0}(z) \underline{b}_{a}+\alpha_{2}(z) \underline{b}_{2}=\operatorname{curl} \underline{a}+\alpha_{2}(z) \underline{b}_{2}$ in case of homogenization with $n=2$.

Equation (10) and the vector extension of (7) give after space and time discretization a system of nonlinear algebraic equations that can be solved by means of the Newton-Raphson method. Deriving the equations with respect to the unknowns (coefficients of basis functions $\alpha_{i}$ and $\underline{\gamma}_{i}$ ) gives rise to the differential reluctivity tensor $\frac{\partial \underline{h}_{f e}}{\partial \underline{b}}$. For isotropic materials with scalar reluctivity $\nu_{f e}(b)$, this tensor can be written as follows:

$$
\frac{\partial \underline{h}_{f e}}{\partial \underline{b}}=\nu_{f e} \underline{\underline{1}}+2 \frac{d \nu_{f e}}{d b^{2}} \underline{b} \underline{b},
$$

where $\underline{1}$ is the unit tensor and $\underline{b} \underline{b}$ the dyadic square of $\underline{b}$. In matrix notation (in 2D case for sake of brevity) this becomes:

$$
\left[\frac{\partial \underline{h}_{f e}}{\partial \underline{b}}\right]=\left[\begin{array}{ll}
\frac{\partial h_{x}}{\partial b_{x}} & \frac{\partial h_{x}}{\partial b_{y}} \\
\frac{\partial h_{y}}{\partial b_{x}} & \frac{\partial h_{y}}{\partial b_{y}}
\end{array}\right]=\nu_{f e}\left[\begin{array}{ll}
1 & 0 \\
0 & 1
\end{array}\right]+2 \frac{d \nu_{f e}}{d b^{2}}\left[\begin{array}{ll}
b_{x} b_{x} & b_{x} b_{y} \\
b_{y} b_{x} & b_{y} b_{y}
\end{array}\right] .
$$

The contribution of $\Omega_{l}$ to the elements of the Jacobian matrix is thus:

$$
\left(\frac{\theta}{d} \int_{-d / 2}^{d / 2} \frac{\partial \underline{h}_{f e}}{\partial \underline{b}}\left\{\begin{array}{c}
\alpha_{0} \operatorname{curl} \underline{\gamma}_{i} \\
\alpha_{2} \underline{\zeta}_{k}
\end{array}\right\},\left\{\begin{array}{c}
\alpha_{0} \operatorname{curl} \underline{\gamma}_{j} \\
\alpha_{2} \underline{\zeta}_{l}
\end{array}\right\} d z\right)_{\Omega_{l}} .
$$

\section{APPLICATION EXAMPLE}

In order to validate the proposed homogenization method, we consider a field problem displaying symmetry with respect to the $x y$-plane, and axisymmetry with respect to the $z$-axis (Fig. 3). The FE model consists of part of a laminated ring core (20 laminations, $d=0.5 \mathrm{~mm}, \sigma=5 \cdot 10^{6} \mathrm{~S} / \mathrm{m}$, separated by $0.02 \mathrm{~mm}$ thick airgaps), a toroidal 100 -turn coil and the air between the ring core and the coil [4]. A nonlinear $\mathrm{BH}$ relation is considered for the iron, viz $\nu_{f e}=h / b=100+$ $10 e^{1.8 b^{2}}$ with $h$ in $\mathrm{A} / \mathrm{m}$ and $b$ in $\mathrm{T}$. The linear case has been treated in [4].

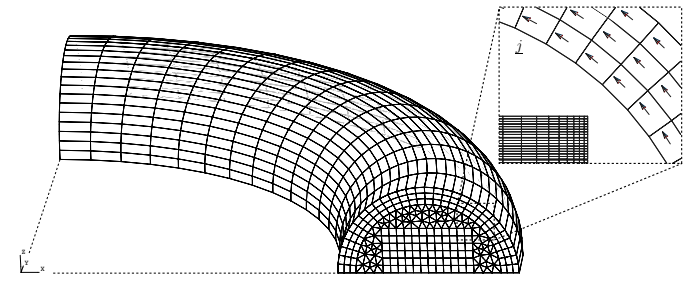

Fig. 3. 1/8th FE model of laminated ring core (discretization of homogenized core) with toroidal coil - zoom: current density in coil and fine discretization of the laminations

Exploiting the symmetry fully, the FE model can be limited to the upper half of the geometry (10 laminations) and a wedge of arbitrary opening angle $\Delta \theta$. Volume meshes of hexahedral and prismatic elements are obtained by circularly extruding (around the $z$-axis, over the angle $\Delta \theta$ in $m$ layers) a 2D mesh of quadrangles and triangles of a radial cross-section (in the $x z$-plane).

The brute-force calculations, with direct inclusion of the eddy currents, are carried out with a fine mesh, in which each of the ten laminations are discretized. A FE model of 1/128th of the complete geometry $(\Delta \theta=2 \pi / 64, m=1,8$ layers of hexahedra per lamination thickness, see zoom in Fig. 3) produces 14406 spatial degrees of freedom for $\underline{a}$.

The homogenization technique is applied to a much coarser mesh, with only 6 layers of hexahedra for the half-thickness of the core (see Fig. 3). For a 1/8th model $(\Delta \theta=2 \pi / 4$ and $m=16)$, this leads a total of 8680 spatial degrees of freedom for $\underline{a}(n=0)$. With the skin effect approximation $n=2$, there are 2304 additional degrees of freedom for $\underline{b}_{2}$.

Time-stepping simulations with imposed sinusoidal current of same amplitude but of different frequencies $f(50 \mathrm{~Hz}$, $250 \mathrm{~Hz}$ and $500 \mathrm{~Hz}$ ) are carried out. In order to reach steadystate, three periods $[0,3 T]$ are time stepped with the backward Euler scheme and $\Delta t=T / 200$. 
The flux linkage of the coil is calculated by integrating $\underline{j} \cdot \underline{a}$ over the coil volume. The flux waveforms obtained with the fine model (reference solution) and the homogenized model ( $n=0$ and $n=2$ ) are compared in Fig. 4. One clearly observes the saturation and the effect of the eddy currents. The homogenization method produces satisfactory results with $n=2$ for all frequencies considered, whereas with $n=0$ it is sufficiently accurate only for the $50 \mathrm{~Hz}$ case.

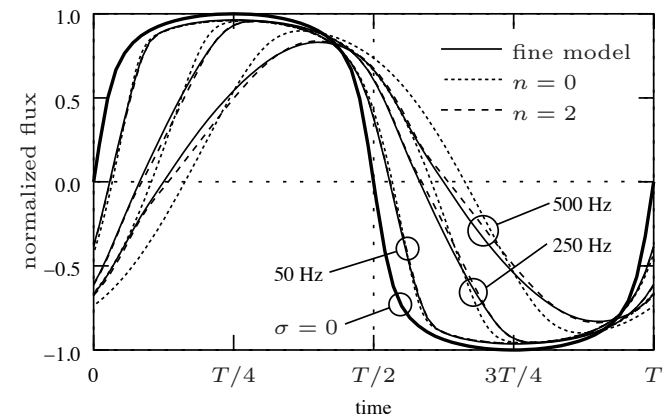

Fig. 4. Normalized flux vs time, calculated with fine model and homogenization method $(n=0$ and $n=2)$

By way of illustration some results obtained with the fine mesh and with the $500 \mathrm{~Hz}$ current are shown in Figs. 5 and 6. The variation of the induction throughout the thickness of the laminations is evidenced in Figs. 5. The circulation of the eddy currents with the $180^{\circ}$-turn at the lamination edge is depicted in Fig. 6.

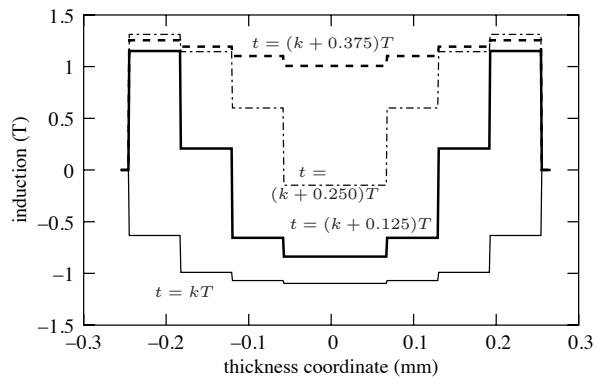

Fig. 5. Variation of induction throughout thickness of lower lamination at $500 \mathrm{~Hz}$, obtained with fine model (4 equidistant instants in half a time period; at average radius)

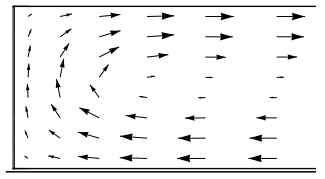

$$
t=k T
$$

$$
\left(j_{\max }=3.4 \mathrm{~A} / \mathrm{mm}^{2}\right)
$$

Fig. 6. Current density in cross-section of lower lamination (near inner radius) at $500 \mathrm{~Hz}$ obtained with fine model

Transient and steady-state waveforms of $b_{a}$ and $b_{2}$ obtained with the homogenization method $(n=2)$ are shown in Figs. 7 and 8 .

\section{CONCLUSION}

A novel homogenization technique for laminated iron cores in $3 \mathrm{D}$ FE models has been proposed. It can be used for

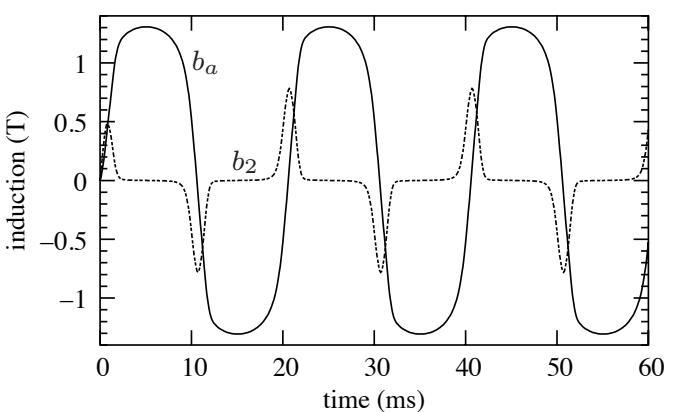

Fig. 7. $50 \mathrm{~Hz}$ induction components $b_{a}(t)$ and $b_{2}(t)$ at average radius (homogenization with $n=2$ )

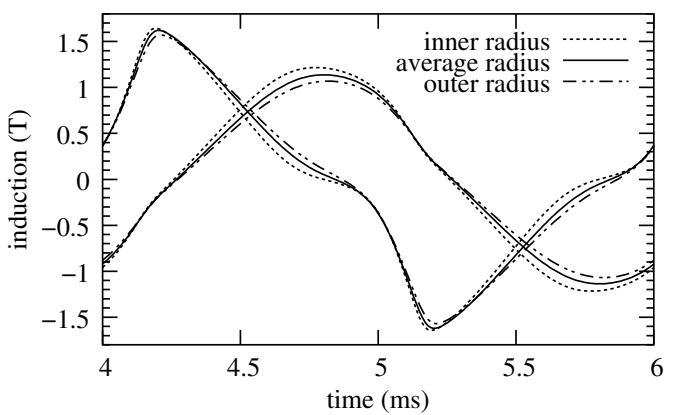

Fig. 8. $\quad 500 \mathrm{~Hz}$ induction components $b_{a}(t)$ and $b_{2}(t)$ at inner, average and outer radius (homogenization with $n=2$ )

time-stepping simulations with the magnetic vector potential formulation. The eddy current effects (including skin effect) in the laminations are taken into account by considering skineffect basis functions and associated degrees of freedom in the homogenized core.

The proposed homogenization method is validated by means a 3D axisymmetric test case. The results agree well with those obtained with a fine model, i.e. in which all laminations are finely discretized and the eddy currents are directly modelled.

\section{REFERENCES}

[1] G. Bertotti, A. Boglietti, M. Chiampi, D. Chiarabaglio, F. Fiorillo and M. Lazzari, "Calculation of eddy currents and associated losses in electrical steel laminations," IEEE Trans. on Magn., Vol. 27, pp. 50075009, Nov. 1991.

[2] J. Gyselinck, L. Vandevelde, J. Melkebeek, P. Dular, F. Henrotte, and W. Legros, "Calculation of eddy currents and associated losses in electrical steel laminations," IEEE Trans. on Magn., Vol. 35, pp. 1191-1194, May 1999

[3] P. Dular, J. Gyselinck, C. Geuzaine, N. Sadowski, and J. P. A. Bastos, "A 3D magnetic vector potential formulation taking eddy currents in lamination stacks into account," IEEE Trans. on Magn., Vol. 39, pp. 14241427, May 2003.

[4] J. Gyselinck and P. Dular, "A time-domain homogenization technique for laminated iron cores in 3D finite element models," IEEE Trans. on Magn., Vol. 40, pp. 1424-1427, May 2004.

[5] J. Lammeraner and M. Štafl, Eddy currents, ILIFFE Books, London, 1966.

[6] L. Dupré, O. Bottauscio, M. Chiampi, M. Repetto and J. Melkebeek, "Modeling of electromagnetic phenomena in soft magnetic materials under unidirectional time periodic flux excitations," IEEE Trans. on Magn., Vol. 35, pp. 4171-4184, Sept. 1999. 